\title{
Influence of Microstructure on the Optical Quality of Silicon Carbide Films: A TEM Study
}

\author{
S. Rajasekhara,* B. Neuner III,** G. Shvets, ** P. J. Ferreira,* D. Kovar,* \\ * Materials Science \& Engineering Program, Cockrell School of Engineering, The University of \\ Texas, Austin, Austin, TX 78712 \\ ** Department of Physics, College of Natural Sciences, The University of Texas, Austin, Austin, \\ TX 78712
}

In this work, we employ transmission electron microscopy (TEM) to understand the microstructure of two silicon carbide ( $\mathrm{SiC}$ ) films (M1 and M2) epitaxially grown on a silicon ( $\mathrm{Si}$ ) substrate, and subsequently correlate our observations with their optical response (transmission and absorbance).

$\mathrm{SiC}$ exhibits a negative permittivity $\left(\varepsilon_{S i C}\right)$ in the mid-infrared (IR) regime, giving it metal-like properties that support plasmon-like surface phonon-polaritons (SPPs). Recently, Urzhumov et al. [1] optimized the size and periodicity of air-bridged $\mathrm{SiC}$ hole arrays supported on a Si substrate and demonstrated that SPPs, which are present in this device, mediate transmission and absorption at tunable IR frequencies. Such properties are desirable for applications such as refractive index and infrared absorption spectroscopy sensing. Clearly, the photonic device efficiency of SiC depends on its optical quality, which is ultimately thought to depend on the crystallinity of SiC and possibly the nature of defects (dislocations and impurities) present. Therefore, understanding the microstructure of $\mathrm{SiC}$ is critical for the successful implementation of these devices in the aforementioned applications.

The TEM results obtained in this work show that the $\mathrm{M} 1 \mathrm{SiC}$ film is a single crystal with a relatively smooth surface (Fig. 1a-b), but with an extensive network of dislocations (Fig. 1c). In contrast, the M2 film has a rough surface and is polycrystalline (Fig. 2a-b), evident from the continuous rings present in the cross-section and plan-view diffraction patterns (Figs. 2b-c). Not surprisingly, the optical data from the two membranes are different. The M1 single crystal film exhibits sharp absoption and transmission lines at approximately $10 \mu \mathrm{m}$ wavelength (Fig. 3a), while the polycrystalline M2 film does not (Fig. 3b). More importantly, the total loss (absorption, scattering) in the single crystal membrane is less than that of the polycrystalline membrane (Fig. 3). Thus, despite their high defect density, the single crystal films seem to be optically superior to the polycrystalline counterpart.

\section{References}

[1] Y. Urzhumov, D. Korobkin, B. Neuner III, C. Zorman, and G. Shvets, J. Opt A. Pure Appl. 9 (2007) S322

[2] This work was supported by the Laboratory Directed Research and Development (LDRD) program at Sandia National Laboratories. Sandia is a multiprogram laboratory operated by Sandia Corporation, a Lockheed Martin Company, for the United States Department of Energy's National Nuclear Security Administration under Contract DE-AC04-94AL85000. 

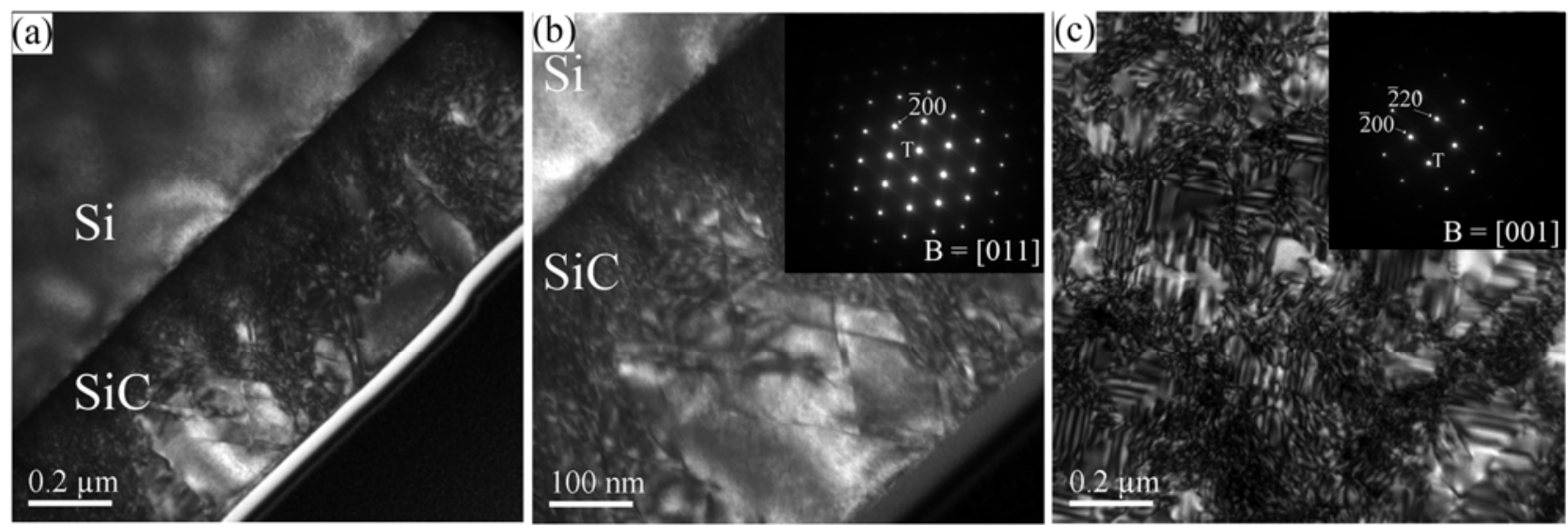

FIG 1: (a) Cross-section image of the M1 film shows a smooth SiC layer approximately $0.52 \mu \mathrm{m}$ thick, (b) diffraction pattern from the SiC layer indicates that it is a single crystal, and (c) plan-view image confirming that $\mathrm{SiC}$ is a single crystal and contains many dislocations.
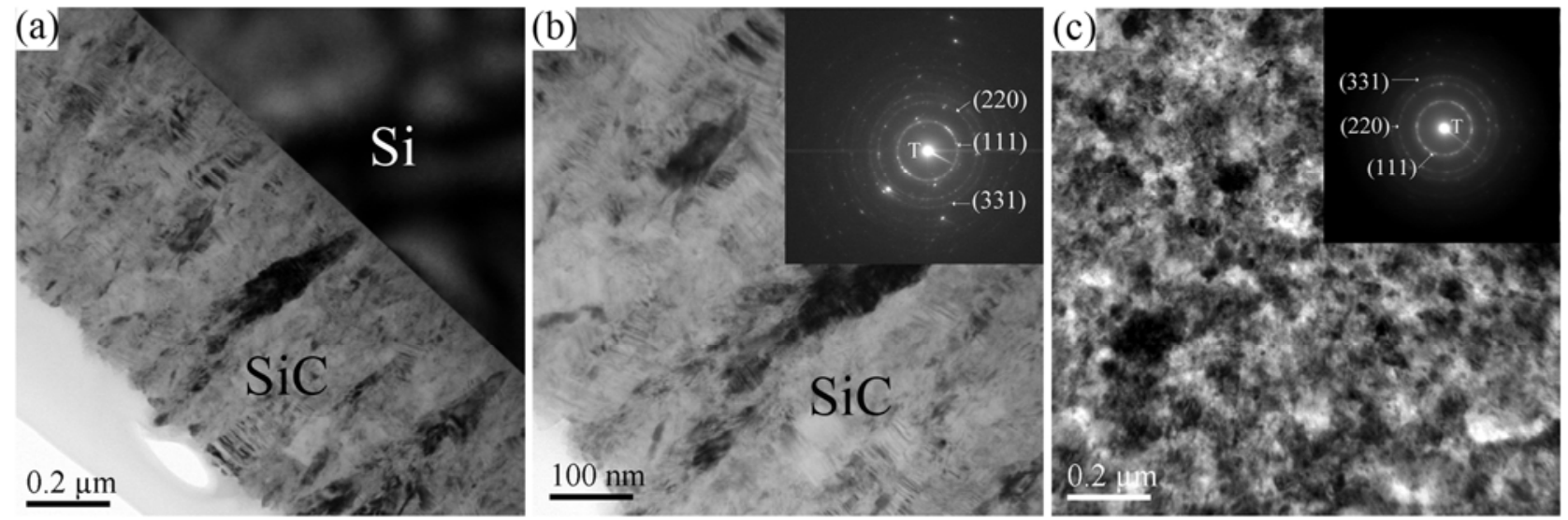

FIG 2: (a) Cross-section image of the M2 film shows a rough $\mathrm{SiC}$ layer approximately $0.58 \mu \mathrm{m}$ thick, (b) diffraction pattern from the SiC layer indicates that it is a polycrystal, and (c) the planview image confirms that $\mathrm{SiC}$ membrane is polycrystalline.
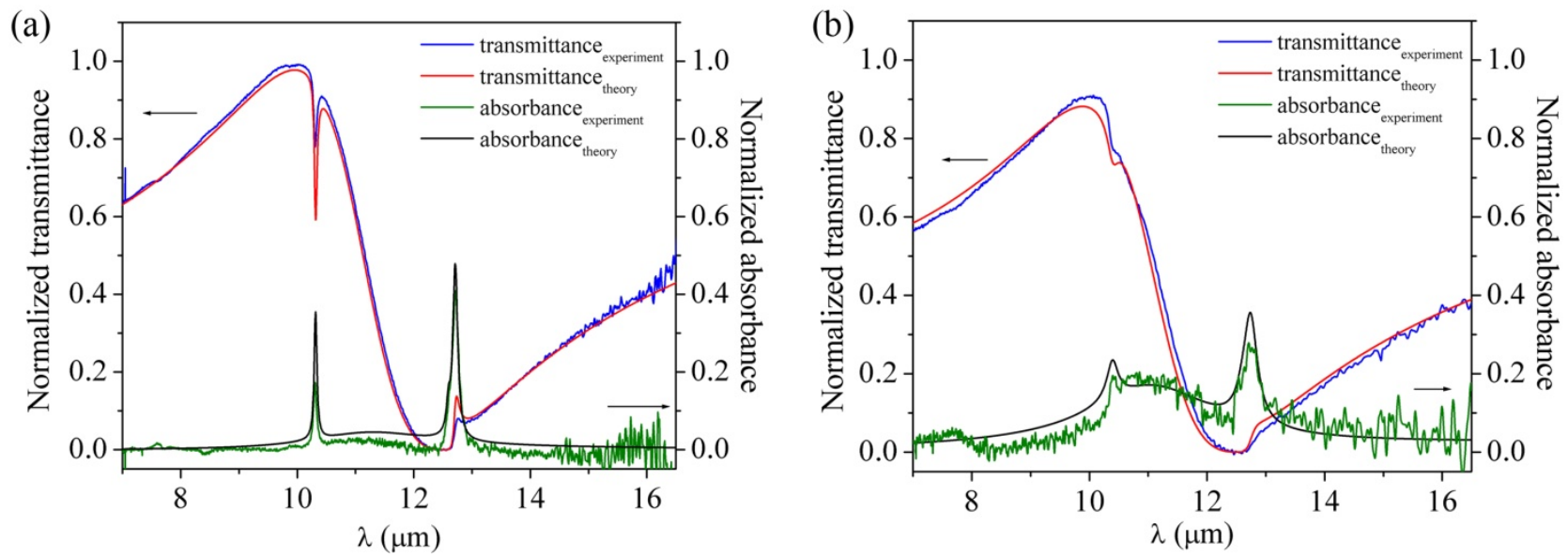

FIG. 3: Optical spectra from the two membranes: (a) the $520 \mathrm{~nm}$-thick M1 membrane exhibits an efficient transmittance and relatively low total loss (absorption and scattering), (b) the $580 \mathrm{~nm}$-thick M2 membrane exhibits a less-efficient transmittance and greater total loss. 